\title{
Effect of vitamins and growth regulators on the vegetative growth of Lentinus squarrosulus
}

\author{
Atri $\mathrm{NS}^{1}$, Kumari $\mathrm{B}^{1}$, Singh $\mathrm{R}^{1}$ and Upadhyay $\mathrm{RC}^{2}$ \\ ${ }^{1}$ Department of Botany, Punjabi University, Patiala \\ ${ }^{2}$ Directorate of Mushroom Research, Chambaghat, Solan, India
}

Atri NS, Kumari B, Singh R, Upadhyay RC 2013 - Effect of vitamins and growth regulators on the vegetative growth of Lentinus squarrosulus. Mycosphere 4(6), 1080-1090, Doi $10.5943 /$ mycosphere/4/6/6

\begin{abstract}
The experiments were carried out to observe the effect of vitamins and growth regulators on the vegetative growth of Lentinus squarrosulus (Mont.) Singer. Out of the vitamins and growth regulators employed, nicotinic acid and gibberellic acid, respectively supported the maximum vegetative growth for this mushroom.
\end{abstract}

Key words - basidiomycetes - edible - Lentinus squarrosulus - mushroom

\section{Introduction}

Lentinus squarrosulus belongs to the Basideomycotina sub-division and to Polyporaceae family, recognized by as many as 40 species the world over (Kirk et al. 2008). It is an edible species of the genus Lentinus that is highly appreciated and has a commercial potential in many countries especially China, Japan and United States, collected from rotten stumps of Juglens regia from Kotla Barog (Himachal Pradesh). The present paper documented the details of in-vitro studies undertaken to investigate the suitability of different vitamins and growth regulators for the mycelial growth of indigenous strain of L. squarrosulus cultured on Potato Dextrose Agar from the wild sample.

\section{Materials and methods}

The culture of L. squarrosulus (Mont.) Singer that was used in present experiment was raised from the indigenous collection made from the dry stem of Juglens regia from Kotla Barog of Sirmour District in Himachal Pradesh during July, 2006 (Fig. No. 1). Morphological observations in the field were made according to the field key to mushroom collector (Atri et al. 2005) and taxonomically identified.

\section{Effect of vitamins}

From the basal media prepared for trace element studies, vitamin impurities were removed by 
adding $5 \mathrm{~g} / \mathrm{l}$ of activated charcoal and autoclaving at 15 psi pressure for 15 minutes and subsequently filtering through Micropore filters $(0.22 \mu \mathrm{m}$ pore size $)$. Stock solution of the vitamins was stored in amber colored bottles so as to avoid their oxidation. Five vitamins with five different concentrations were tried to study the effect on mycelial growth (Table 1). In addition to the mixture of all five vitamins, one check with no vitamin was also maintained. The stock solutions of all vitamins except biotin, were prepared in double glass distilled water and stored at $2-5^{0} \mathrm{C}$ in refrigerator. The stock solution of biotin was prepared in $5 \mathrm{ml}$ of 50 per cent ethanol and volume made up with double glass distilled water. Vitamins were sterilized by filtration through Micropore filters $(0.22 \mu \mathrm{m}$ pore size $)$.

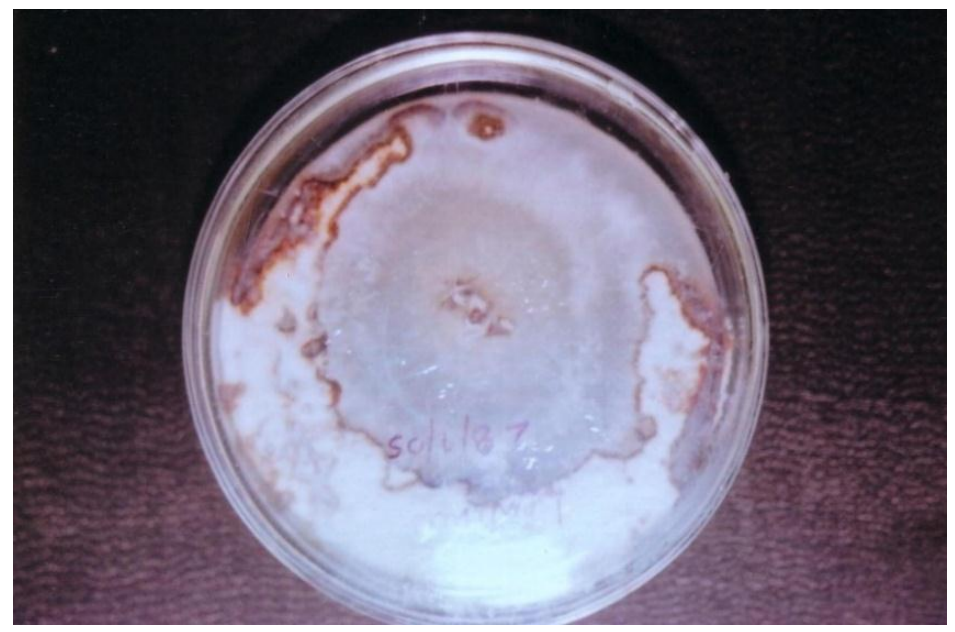

Fig. 1 - Pure culture of Lentinus squarrosulus on Potato dextrose agar

Table 1 The vitamins along with their concentrations used

\begin{tabular}{ll}
\hline Vitamins & Concentrations (ppm) \\
\hline Riboflavin & $10,20,30,40,50$ \\
Nicotinic Acid & $10,20,30,40,50$ \\
Biotin & $10,20,30,40,50$ \\
Ascorbic Acid & $10,20,30,40,50$ \\
Folic Acid & $10,20,30,40,50$ \\
\hline
\end{tabular}

(ii) Effect of growth regulators: The stock solutions of all growth regulators except gibberellic acid (GA) were prepared in double glass distilled water and stored at $5^{0} \mathrm{C}$ in refrigerator (Table 2). GA was first dissolved in $10 \mathrm{ml}$ of acetone and then required dilutions were prepared. Different concentrations of growth regulators used were as follows:-

Table 2 The growth regulators along with their concentrations used are given below:-

\begin{tabular}{ll}
\hline Growth Regulators & Concentrations (ppm) \\
\hline Gibberellic Acid (GA) & $5,10,15,20$ \\
Indole Acetic Acid (IAA) & $5,10,15,20$ \\
Indole Butyric Acid (IBA) & $5,10,15,20$ \\
Kinetin (K) & $5,10,15,20$ \\
Nephthyl Acetic Acid (NAA) & $5,10,15,20$ \\
\hline
\end{tabular}




\section{(i) Evaluation of different vitamins for mycelial growth}

At $10 \mathrm{ppm}$ concentration, maximum mycelial growth of $15.45 \mathrm{mg} / \mathrm{ml}$ was recorded in nicotinic acid followed by $15.25 \mathrm{mg} / \mathrm{ml}$ mycelial growth in the mixture medium where least mycelial growth of $13.75 \mathrm{mg} / \mathrm{ml}$ was supported by folic acid. At $20 \mathrm{ppm}$ concentration maximum mycelial growth of $15.50 \mathrm{mg} / \mathrm{ml}$ was again recorded in nicotinic acid followed by $15.35 \mathrm{mg} / \mathrm{ml}$ mycelial growth in the mixture medium where least mycelial growth of $14.15 \mathrm{mg} / \mathrm{ml}$ was supported by biotin. At $30 \mathrm{ppm}$ concentration maximum mycelial growth of $15.50 \mathrm{mg} / \mathrm{ml}$ was recorded in nicotinic acid followed by $15.45 \mathrm{mg} / \mathrm{ml}$ mycelial growth in the mixture medium where least mycelial growth of $14.20 \mathrm{mg} / \mathrm{ml}$ was supported by biotin. At $40 \mathrm{ppm}$ concentration maximum mycelial growth of $15.75 \mathrm{mg} / \mathrm{ml}$ was recorded in nicotinic acid followed by $15.50 \mathrm{mg} / \mathrm{ml}$ mycelial growth in the mixture medium where least mycelial growth of $15.00 \mathrm{mg} / \mathrm{ml}$ was supported by biotin. At $50 \mathrm{ppm}$ concentration maximum mycelial growth of $15.95 \mathrm{mg} / \mathrm{ml}$ was recorded in nicotinic acid followed by $15.50 \mathrm{mg} / \mathrm{ml}$ mycelial growth in the mixture medium where least mycelial growth of $15.15 \mathrm{mg} / \mathrm{ml}$ was supported by biotin and only 13.70 $\mathrm{mg} / \mathrm{ml}$ mycelial dry weight was recorded in control. Mean dry weight of mycelium in different vitamins along with \pm S.D. are depicted in Table-3 and histogram (Fig-2).

Table 3 Evaluation of impact of variable concentrations of vitamins in the basal medium on the mycelial growth of $L$. squarrosulus.

\begin{tabular}{|c|c|c|c|c|c|c|}
\hline \multirow{2}{*}{$\begin{array}{l}\text { Sr. } \\
\text { No. }\end{array}$} & \multirow{2}{*}{ Vitamin } & \multicolumn{4}{|c|}{ Mean Dry Weight $(\mathrm{mg} / \mathrm{ml}) \pm$ S.D. at variable concentrations } & \multirow[b]{2}{*}{50 ppm } \\
\hline & & 10 ppm & 20 ppm & 30 ppm & 40 ppm & \\
\hline$\overline{1}$ & Riboflavin & $14.30 \pm 1.73$ & $14.75 \pm 2.12$ & $14.80 \pm 1.00$ & $15.20 \pm 1.53$ & $15.25 \pm 1.41$ \\
\hline 2 & Nicotinic acid & $15.45 \pm 0.71$ & $15.50 \pm 2.00$ & $15.50 \pm 1.15$ & $15.75 \pm 1.00$ & $15.95 \pm 1.00$ \\
\hline 3 & Biotin & $14.75 \pm 0.58$ & $14.15 \pm 1.15$ & $14.20 \pm 2.83$ & $15.00 \pm 2.83$ & $15.15 \pm 1.53$ \\
\hline 4 & Ascorbic acid & $14.75 \pm 1.41$ & $15.00 \pm 2.83$ & $15.30 \pm 1.15$ & $15.30 \pm 1.53$ & $15.30 \pm 1.53$ \\
\hline 5 & Folic acid & $13.75 \pm 0.71$ & $14.30 \pm 1.53$ & $14.55 \pm 2.12$ & $15.15 \pm 1.41$ & $15.20 \pm 1.73$ \\
\hline 6 & Mixture & $15.25 \pm 1.41$ & $15.35 \pm 2.12$ & $15.45 \pm 1.73$ & $15.50 \pm 1.15$ & $15.50 \pm 1.15$ \\
\hline 7 & Control & $12.70 \pm 1.00$ & $12.80 \pm 2.83$ & $13.05 \pm 2.00$ & $13.15 \pm 1.73$ & $13.70 \pm 1.41$ \\
\hline
\end{tabular}

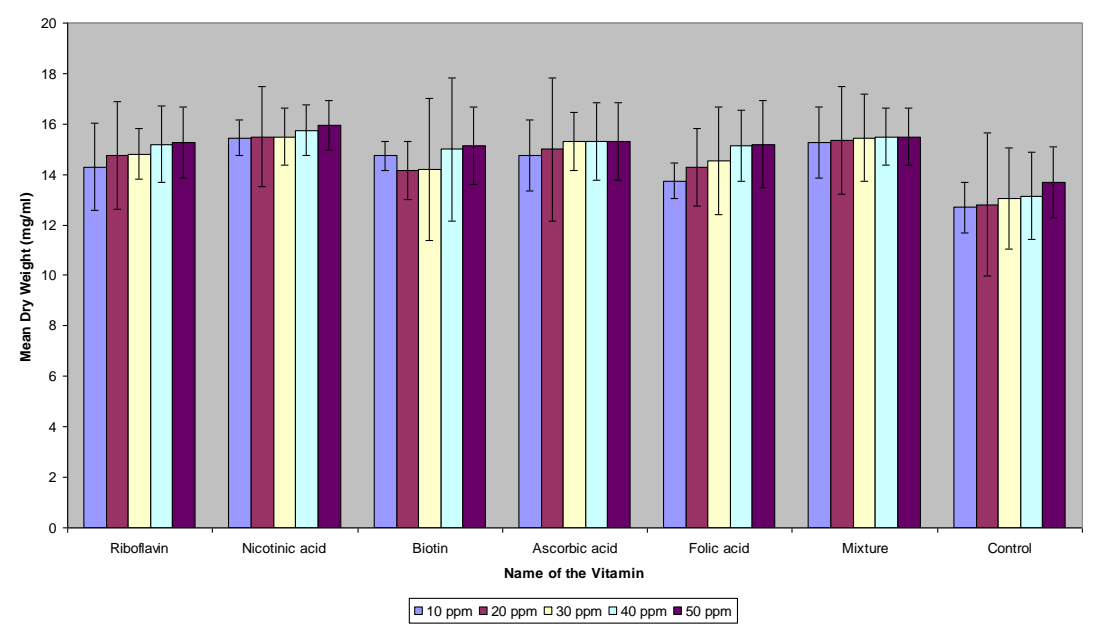

Fig. 2 - Histogram showing effect of different concentration of vitamins for mycelial growth of $L$. squarrosulus. 
At $10 \mathrm{ppm}$ concentration, as per t-values calculated, maximum vegetative growth was supported by nicotinic acid which was calculated significantly higher than growth with biotin $(\mathrm{t}=0.0678, \mathrm{df}=6, \mathrm{p}<0.05)$, ascorbic acid $(\mathrm{t}=0.0125, \mathrm{df}=6, \mathrm{p}<0.05)$, folic acid $(\mathrm{t}=0.0478, \mathrm{df}=6, \mathrm{p}<0.05)$, and control $(\mathrm{t}=0.0634, \mathrm{df}=6, \mathrm{p}<0.05)$ whereas in comparison the growth in the mixture was found to be non significant (Table-4).

Table $4 \mathrm{t}$-value showing the effect of $10 \mathrm{ppm}$ concentration of different vitamins on mycelial growth of L. squarrosulus.

\begin{tabular}{|c|c|c|c|c|c|c|c|c|c|}
\hline Sr. No. & Vitamin & $\begin{array}{l}\text { Dry wt. } \\
(\mathrm{mg} / \mathrm{ml})\end{array}$ & $\begin{array}{l}\mathbf{1} \\
\text { Riboflavin } \\
14.30\end{array}$ & $\begin{array}{l}\mathbf{2} \\
\text { Nicotinic acid } \\
15.45\end{array}$ & $\begin{array}{l}3 \\
\text { Biotin } \\
14.75\end{array}$ & $\begin{array}{l}\mathbf{4} \\
\text { Ascorbic acid } \\
14.75\end{array}$ & $\begin{array}{l}\mathbf{5} \\
\text { Folic acid } \\
13.75\end{array}$ & $\begin{array}{l}\mathbf{6} \\
\text { Mixture } \\
15.25\end{array}$ & $\begin{array}{l}7 \\
\text { Control } \\
12.70\end{array}$ \\
\hline 1 & Riboflavin & $\overline{14.30}$ & -- & $0.0173^{*}$ & $0.0162 *$ & 0.0057 & 0.0083 & $0.0120^{*}$ & $0.0226^{*}$ \\
\hline 2 & Nicotinic acid & 15.45 & & -- & $0.0678^{*}$ & $0.0125 *$ & $0.0478 *$ & 0.0035 & $0.0634 *$ \\
\hline 3 & Biotin & 14.75 & & & -- & 0.0027 & 0.0015 & 0.0037 & $0.0134 *$ \\
\hline 4 & Ascorbic acid & 14.75 & & & & -- & 0.0179* & 0.0070 & 0.0033 \\
\hline 5 & Folic acid & 13.75 & & & & & -- & 0.0026 & 0.0024 \\
\hline 6 & Mixture & 15.25 & & & & & & -- & 0.0041 \\
\hline 7 & Control & 12.70 & & & & & & & -- \\
\hline
\end{tabular}

* Significant at 0.05 level

Table 5 t-values showing the effect of $20 \mathrm{ppm}$ concentration of different vitamins on mycelial growth of L. squarrosulus.

\begin{tabular}{|c|c|c|c|c|c|c|c|c|c|}
\hline $\begin{array}{l}\text { Sr. } \\
\text { No. }\end{array}$ & Vitamin & $\begin{array}{l}\text { Dry wt. } \\
\text { (mg/ml) }\end{array}$ & $\begin{array}{l}\mathbf{1} \\
\text { Riboflavin } \\
14.75\end{array}$ & $\begin{array}{l}2 \\
\text { Nicotinic acid } \\
15.50\end{array}$ & $\begin{array}{l}3 \\
\text { Biotin } \\
14.15\end{array}$ & $\begin{array}{l}\mathbf{4} \\
\text { Ascorbic acid } \\
15.00\end{array}$ & $\begin{array}{l}\mathbf{5} \\
\text { Folic acid } \\
14.30\end{array}$ & $\begin{array}{l}\mathbf{6} \\
\text { Mixture } \\
15.35\end{array}$ & $\begin{array}{l}7 \\
\text { Control } \\
12.80\end{array}$ \\
\hline 1 & Riboflavin & 14.75 & $\overline{--}$ & 0.0072 & 0.0070 & 0.0019 & 0.0048 & 0.0056 & $0.0155^{*}$ \\
\hline 2 & Nicotinic acid & 15.50 & & -- & $0.0165^{*}$ & 0.0040 & $0.0134 *$ & 0.0014 & $0.0220 *$ \\
\hline 3 & Biotin & 14.15 & & & -- & 0.0078 & 0.0022 & $0.0140 *$ & $0.0124 *$ \\
\hline 4 & Ascorbic acid & 15.00 & & & & -- & 0.0061 & 0.0027 & $0.0155^{*}$ \\
\hline 5 & Folic acid & 14.30 & & & & & -- & $0.0113^{*}$ & $0.0131 *$ \\
\hline 6 & Mixture & 15.35 & & & & & & -- & $0.0203 *$ \\
\hline 7 & Control & 12.80 & & & & & & & -- \\
\hline
\end{tabular}

* Significant at 0.05 level

Next best vegetative growth was supported by mixture of all the five vitamins which was significantly higher than growth in riboflavin $(\mathrm{t}=0.0120, \mathrm{df}=6, \mathrm{p}<0.05)$. In comparison, the growth with nicotinic acid, ascorbic acid, folic acid and biotin was found to be non significant (Table-4).

Maximum vegetative growth at $20 \mathrm{ppm}$ was supported by nicotinic acid which was significantly higher than growth with biotin $(\mathrm{t}=0.0165, \mathrm{df}=6, \mathrm{p}<0.05)$, folic acid $(\mathrm{t}=0.0134, \mathrm{df}=6$, $\mathrm{p}<0.05)$, and control $(\mathrm{t}=0.0220, \mathrm{df}=6, \mathrm{p}<0.05)$ whereas in comparison the growth with ascorbic acid and mixture was found to be non significant (Table-5).

Next best results with respect to vegetative growth was supported by mixture of all the five vitamins which was significantly higher than growth in control $(\mathrm{t}=0.0203, \mathrm{df}=6, \mathrm{p}<0.05)$. Least vegetative growth was recorded in biotin (Table-5).

At $30 \mathrm{ppm}$ concentration also maximum vegetative growth was supported by nicotinic acid which was significantly higher than growth achieved in folic acid $(\mathrm{t}=0.0111, \mathrm{df}=6, \mathrm{p}<0.05)$, and control $(\mathrm{t}=0.0300, \mathrm{df}=6, \mathrm{p}<0.05)$ whereas in comparison the growth with biotin, ascorbic acid and mixture was found to be non significant (Table-6). 
Table $6 \mathrm{t}$-values showing the effect of $30 \mathrm{ppm}$ concentration of different vitamins on mycelial growth of L. squarrosulus

\begin{tabular}{|c|c|c|c|c|c|c|c|c|c|}
\hline \multirow[t]{2}{*}{$\begin{array}{l}\text { Sr. } \\
\text { No. }\end{array}$} & Vitamin & & $\begin{array}{l}1 \\
\text { Riboflavin }\end{array}$ & $\begin{array}{l}2 \\
\text { Nicotinic acid }\end{array}$ & $\begin{array}{l}3 \\
\text { Biotin }\end{array}$ & $\begin{array}{l}4 \\
\text { Ascorbic acid }\end{array}$ & $\begin{array}{l}5 \\
\text { Folic acid }\end{array}$ & $\begin{array}{l}6 \\
\text { Mixture }\end{array}$ & $\begin{array}{l}7 \\
\text { Control }\end{array}$ \\
\hline & & $\begin{array}{l}\text { Dry wt. } \\
(\mathrm{mg} / \mathrm{ml})\end{array}$ & 14.80 & 15.50 & 14.20 & 15.30 & 14.55 & 15.45 & 13.05 \\
\hline 1 & Riboflavin & $\overline{14.80}$ & -- & $0.0129 *$ & $0.0565^{*}$ & 0.0092 & 0.0030 & 0.0092 & $0.0221^{*}$ \\
\hline 2 & Nicotinic acid & 15.50 & & -- & 0.0069 & 0.0034 & $0.0111^{*}$ & 0.0006 & $0.0300 *$ \\
\hline 3 & Biotin & 14.20 & & & -- & $0.0101 *$ & 0.0027 & $0.0106 *$ & 0.0093 \\
\hline 4 & Ascorbic acid & 15.30 & & & & -- & 0.0087 & 0.0020 & $0.0275^{*}$ \\
\hline 5 & Folic acid & 14.55 & & & & & -- & 0.0093 & $0.0145^{*}$ \\
\hline 6 & Mixture & 15.45 & & & & & & -- & $0.0256^{*}$ \\
\hline 7 & Control & 13.05 & & & & & & & -- \\
\hline
\end{tabular}

* Significant at 0.05 level

At this concentration the next best vegetative growth was supported by mixture of all the five vitamins which was significantly higher than growth in control $(\mathrm{t}=0.0256, \mathrm{df}=6, \mathrm{p}<0.05)$. Least vegetative growth was recorded in biotin (Table-6).

As per the t-values obtained, the maximum vegetative growth at $40 \mathrm{ppm}$ concentration was supported by nicotinic acid in the basal medium which was significantly higher than growth in control $(\mathrm{t}=0.0368, \mathrm{df}=6, \mathrm{p}<0.05)$. In comparison the growth with biotin, ascorbic acid, folic acid and mixture was found to be non significant (Table-7).

Second maximum vegetative growth was supported by mixture of all the five vitamins which was significantly higher than growth in control $(\mathrm{t}=0.0319, \mathrm{df}=6, \mathrm{p}<0.05)$. Least vegetative growth was recorded in biotin (Table-7).

Table $7 \mathrm{t}$-values showing the effect of $40 \mathrm{ppm}$ concentration of different vitamins on mycelial growth of L. squarrosulus.

\begin{tabular}{|c|c|c|c|c|c|c|c|c|c|}
\hline \multirow[t]{2}{*}{$\begin{array}{l}\text { Sr. } \\
\text { No. }\end{array}$} & Vitamin & & $\begin{array}{l}1 \\
\text { Riboflavin }\end{array}$ & $\begin{array}{l}2 \\
\text { Nicotinic acid }\end{array}$ & $\begin{array}{l}3 \\
\text { Biotin }\end{array}$ & $\begin{array}{l}4 \\
\text { Ascorbic acid }\end{array}$ & $\begin{array}{l}5 \\
\text { Folic acid }\end{array}$ & $\begin{array}{l}6 \\
\text { Mixture }\end{array}$ & $\begin{array}{l}7 \\
\text { Control }\end{array}$ \\
\hline & & $\begin{array}{l}\text { Dry wt } \\
(\mathrm{mg} / \mathrm{ml})\end{array}$ & 15.20 & 15.75 & 15.00 & 15.30 & 15.15 & 15.50 & 13.15 \\
\hline 1 & Riboflavin & 15.20 & -- & 0.0085 & 0.0017 & 0.0013 & 0.0006 & 0.0044 & $0.0251 *$ \\
\hline 2 & Nicotinic acid & 15.75 & & -- & 0.0070 & 0.0069 & 0.0098 & 0.0046 & $0.0368 *$ \\
\hline 3 & Biotin & 15.00 & & & -- & 0.0026 & 0.0013 & 0.0046 & $0.0157 *$ \\
\hline 4 & Ascorbic acid & 15.30 & & & & -- & 0.0020 & 0.0029 & $0.0263 *$ \\
\hline 5 & Folic acid & 15.15 & & & & & -- & $0.0544 *$ & $0.0253 *$ \\
\hline 6 & Mixture & 15.50 & & & & & & -- & $0.0319 *$ \\
\hline 7 & Control & 13.15 & & & & & & & -- \\
\hline
\end{tabular}

At $50 \mathrm{ppm}$ concentration again maximum vegetative growth was supported by nicotinic acid which was significantly higher than growth in biotin $(\mathrm{t}=0.0123, \mathrm{df}=6, \mathrm{p}<0.05)$, mixture $(\mathrm{t}=0.0835$, $\mathrm{df}=6, \mathrm{p}<0.05)$ and control $(\mathrm{t}=0.0368, \mathrm{df}=6, \mathrm{p}<0.05)$ whereas in comparison the growth with ascorbic acid and folic acid was found to be non significant (Table-8).

Next best vegetative growth was supported by mixture of all the five vitamins which was significantly higher than growth in control $(\mathrm{t}=0.0279, \mathrm{df}=6, \mathrm{p}<0.05)$. Least vegetative growth was recorded in biotin (Table-8). 
Table $8 \mathrm{t}$-values showing the effect of $50 \mathrm{ppm}$ concentration of different vitamins on mycelial growth of L. squarrosulus.

\begin{tabular}{|c|c|c|c|c|c|c|c|c|c|}
\hline $\begin{array}{l}\text { Sr. } \\
\text { No }\end{array}$ & Vitamin & & $\begin{array}{l}1 \\
\text { Riboflavin }\end{array}$ & $\begin{array}{l}2 \\
\text { Nicotinic acid }\end{array}$ & $\begin{array}{l}3 \\
\text { Biotin }\end{array}$ & $\begin{array}{l}4 \\
\text { Ascorbic acid }\end{array}$ & $\begin{array}{l}5 \\
\text { Folic acid }\end{array}$ & $\begin{array}{l}6 \\
\text { Mixture }\end{array}$ & $\begin{array}{l}7 \\
\text { Control }\end{array}$ \\
\hline & & $\begin{array}{l}\text { Dry } \\
\text { wt.(mg/ml) }\end{array}$ & 15.25 & 15.95 & 15.15 & 15.30 & 15.20 & 15.50 & 13.70 \\
\hline 1 & Riboflavin & 15.25 & -- & $0.0114^{*}$ & 0.0013 & 0.0006 & 0.0006 & 0.0038 & $0.0219^{*}$ \\
\hline 2 & Nicotinic acid & 15.95 & & -- & $0.0123^{\prime}$ & 0.0010 & 0.0010 & $0.0835^{*}$ & $0.0368 *$ \\
\hline 3 & Biotin & 15.15 & & & -- & 0.0019 & 0.0006 & $0.0517 *$ & $0.0917 *$ \\
\hline 4 & Ascorbic acid & 15.30 & & & & -- & $0.0122^{*}$ & 0.0029 & $0.0217 *$ \\
\hline 5 & Folic acid & 15.20 & & & & & -- & 0.0040 & $0.0190^{*}$ \\
\hline 6 & Mixture & 15.50 & & & & & & -- & $0.0279 *$ \\
\hline 7 & Control & 13.70 & & & & & & & -- \\
\hline
\end{tabular}

* Significant at 0.05 level

\section{(ii) Evaluation of different growth regulators for mycelial growth}

So as to evaluate the effect of variable concentrations of growth regulators in the basal medium, five different growth regulators namely 1-napthyl acetic acid (NAA), indole 3-acetic acid (IAA), gibberellic acid (GA), kinetin (K) and indole-3-butyric acid (IBA) with concentrations of 5 ppm, 10 ppm, $15 \mathrm{ppm}$, and $20 \mathrm{ppm}$ along with the mixture of all the five growth regulators were selected. Yeast glucose medium with $\mathrm{pH} 4.0$ was taken as the basal medium. The medium flasks were inoculated with freshly homogenized inoculum and then respective growth regulators were added through micropore filters. Then these inoculated flasks were incubated at $30 \pm 1^{0} \mathrm{C}$ for 13 days and mycelial dry weight was recorded in milligrams. At $5 \mathrm{ppm}$ concentration, maximum mycelial growth of $16.05 \mathrm{mg} / \mathrm{ml} \mathrm{was}$ recorded in gibberellic acid followed by $15.25 \mathrm{mg} / \mathrm{ml}$ mycelial growth in NAA where least mycelial growth of $11.75 \mathrm{mg} / \mathrm{ml}$ was supported by IBA. At $10 \mathrm{ppm}$ concentration maximum mycelial growth of $16.50 \mathrm{mg} / \mathrm{ml}$ was recorded in gibberellic acid followed by $15.50 \mathrm{mg} / \mathrm{ml}$ mycelial growth in NAA where least mycelial growth of $11.85 \mathrm{mg} / \mathrm{ml}$ was supported by IBA. At $15 \mathrm{ppm}$ concentration maximum mycelial growth of $16.75 \mathrm{mg} / \mathrm{ml}$ was recorded in gibberellic acid followed by $15.90 \mathrm{mg} / \mathrm{ml}$ mycelial growth in NAA where least mycelial growth of $12.20 \mathrm{mg} / \mathrm{ml}$ was supported by IBA. At 20 ppm concentration maximum mycelial growth of $17.55 \mathrm{mg} / \mathrm{ml}$ was recorded in gibberellic acid followed by $16.20 \mathrm{mg} / \mathrm{ml}$ mycelial growth in NAA where least mycelia growth of $12.45 \mathrm{mg} / \mathrm{ml} \mathrm{was}$ supported by IBA and only $12.30 \mathrm{mg} / \mathrm{ml}$ mycelial dry weight was recorded in control. Mean dry weight of the mycelium in different growth regulators along with \pm S.D. are depicted in Table-9 and histogram (Fig-3).

Table 9 Effect of variable concentration of growth regulators on mycelial growth of L. squarrosulus.

\begin{tabular}{|c|c|c|c|c|c|}
\hline$\overline{\text { Sr. }}$ & Growth Regulator & Mean Dry $\mathrm{M}$ & $(\mathrm{mg} / \mathrm{ml}) \pm \mathrm{S}$ & at variable c & ntrations \\
\hline No. & & $5 \mathrm{ppm}$ & $10 \mathrm{ppm}$ & 15 ppm & 20 ppm \\
\hline 1 & 1-Nepthyl Acetic Acid & $15.25 \pm 1.53$ & $15.50 \pm 1.00$ & $15.9 \pm 1.41$ & $16.20 \pm 1.41$ \\
\hline 2 & Indole Acetic Acid & $12.65 \pm 0.71$ & $12.80 \pm 2.52$ & $13.25 \pm 0.58$ & $13.55 \pm 1.15$ \\
\hline 3 & Gibberellic Acid & $16.05 \pm 1.73$ & $16.50 \pm 2.83$ & $16.75 \pm 1.15$ & $17.55 \pm 2.31$ \\
\hline 4 & Kinetin & $14.20 \pm 0.71$ & $14.65 \pm 1.15$ & $15.30 \pm 1.41$ & $15.55 \pm 1.00$ \\
\hline 5 & Indole Butyric Acid & $11.75 \pm 1.41$ & $11.85 \pm 0.58$ & $12.20 \pm 1.71$ & $12.45 \pm 2.65$ \\
\hline 6 & Mixture & $15.15 \pm 1.21$ & $15.25 \pm 1.00$ & $15.60 \pm 0.63$ & $15.90 \pm 1.21$ \\
\hline 7 & Control & $11.55 \pm 1.15$ & $12.00 \pm 2.12$ & $12.15 \pm 2.31$ & $12.30 \pm 1.53$ \\
\hline
\end{tabular}

Maximum vegetative growth at $5 \mathrm{ppm}$ concentration was supported by gibberellic acid which was significantly higher than growth in kinetin $(\mathrm{t}=0.0279, \mathrm{df}=6, \mathrm{p}<0.05)$, indole butyric acid $(\mathrm{t}=0.0544$, $\mathrm{df}=6, \mathrm{p}<0.05)$, Mixture $(\mathrm{t}=0.0369, \mathrm{df}=6, \mathrm{p}<0.05)$ and control $(\mathrm{t}=0.0612, \mathrm{df}=6, \mathrm{p}<0.05)($ Table-10). 
Next best vegetative growth was supported by nephthyl acetic acid which has been recorded to be significantly higher than growth in IAA $(\mathrm{t}=0.0435, \mathrm{df}=6, \mathrm{p}<0.05)$, kinetin $(\mathrm{t}=0.0176, \mathrm{df}=6$, $\mathrm{p}<0.05)$, indole butyric acid $(\mathrm{t}=0.0475, \mathrm{df}=6, \mathrm{p}<0.05)$, mixture $(\mathrm{t}=0.0357, \mathrm{df}=6, \mathrm{p}<0.05)$ and Control $(\mathrm{t}=0.0546, \mathrm{df}=6, \mathrm{p}<0.05)$. In comparison the growth with gibberellic acid was found to be non significant. Least vegetative growth was recorded in indole butyric acid (Table-8) which was significantly higher than growth in mixture $(\mathrm{t}=0.0239, \mathrm{df}=6, \mathrm{p}<0.05)$. In comparison the growth in Control was found to be non significant.

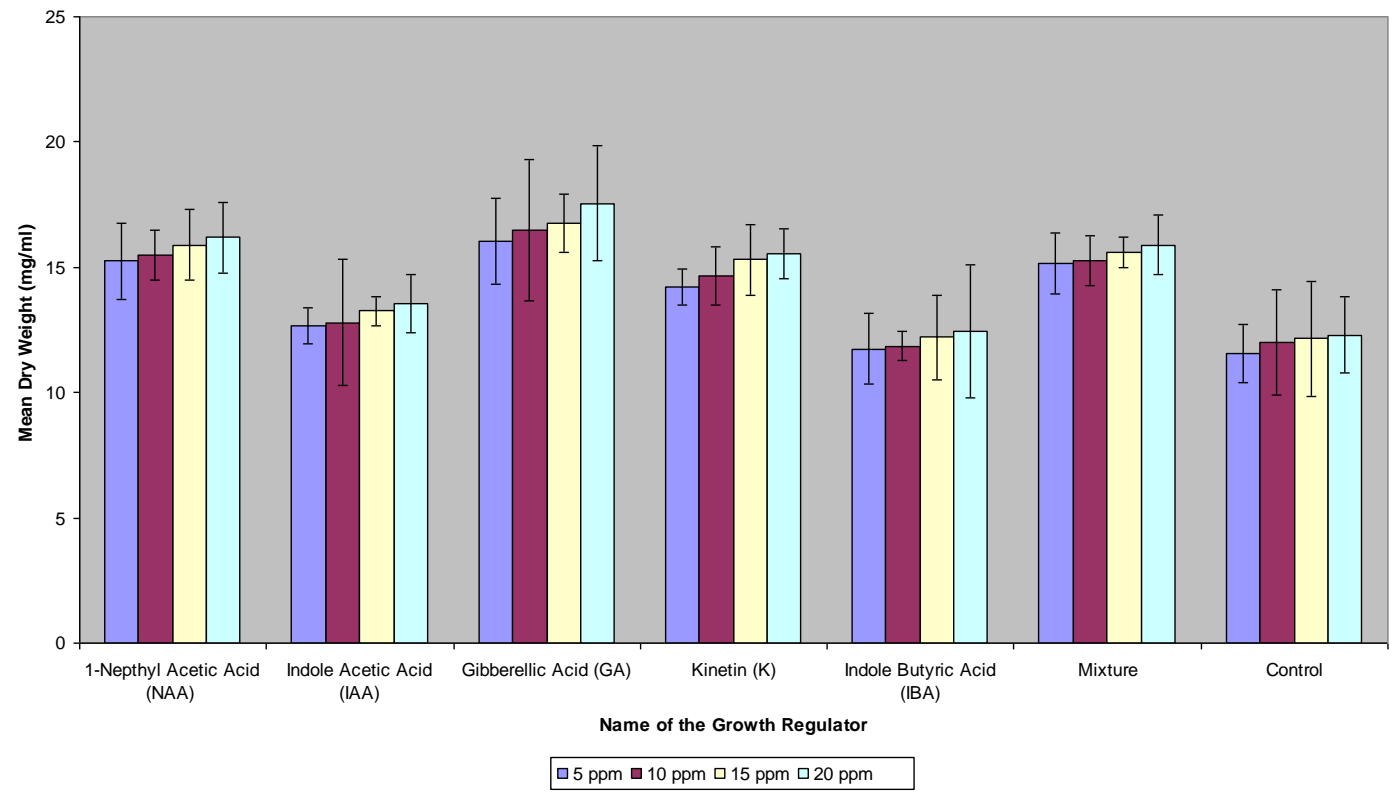

Fig. 3- Histogram showing effect of different concentration of growth regulators for mycelial growth of L. squarrosulus.

Table 10 t-value showing the effect of 5 ppm concentration of different growth regulators on mycelial growth of L. squarrosulus.

\begin{tabular}{|c|c|c|c|c|c|c|c|c|c|}
\hline \multirow{2}{*}{$\begin{array}{l}\bar{S} \\
\mathbf{N}\end{array}$} & & & 1 & & 3 & \multirow{2}{*}{$\begin{array}{l}4 \\
\text { Kinetin }\end{array}$} & \multirow{2}{*}{$\begin{array}{l}5 \\
\text { Indole Butyric } \\
\text { Acid }\end{array}$} & \multirow{2}{*}{ Mixture } & \multirow{2}{*}{$\begin{array}{l}7 \\
\text { Control }\end{array}$} \\
\hline & Growth regulator & & $\begin{array}{c}\text { Nepthyl Acetic } \\
\text { Acid }\end{array}$ & $\begin{array}{c}\text { Indole Acetic } \\
\text { Acid }\end{array}$ & $\begin{array}{c}\text { Gibberellic } \\
\text { Acid }\end{array}$ & & & & \\
\hline & & $\begin{array}{l}\text { Dry wt. } \\
(\mathrm{mg} / \mathrm{ml})\end{array}$ & 15.25 & 12.65 & 16.05 & 14.20 & 11.75 & 15.15 & 11.55 \\
\hline 1 & Nepthyl Acetic Acid & 15.25 & -- & $0.0435^{*}$ & 0.0097 & $0.0176^{*}$ & $0.0475 *$ & $0.0357 *$ & $0.0546^{*}$ \\
\hline 2 & Indole Acetic Acid & 12.65 & & -- & $0.0514 *$ & $0.0436 *$ & $0.0161 *$ & $0.0101 *$ & $0.0230 *$ \\
\hline 3 & Gibberellic Acid & 16.05 & & & -- & $0.0279 *$ & $0.0544 *$ & $0.0369 *$ & $0.0612 *$ \\
\hline 4 & Kinetin & 14.20 & & & & -- & $0.0438 *$ & $0.0397 *$ & $0.0554 *$ \\
\hline 5 & Indole Butyric Acid & 11.75 & & & & & -- & $0.0239 *$ & 0.0031 \\
\hline 6 & Mixture & 15.15 & & & & & & -- & $0.0316^{*}$ \\
\hline 7 & Control & 11.55 & & & & & & & -- \\
\hline
\end{tabular}

* Significant at 0.05 level

Maximum vegetative growth at $10 \mathrm{ppm}$ concentration was recorded in gibberellic acid which was significantly higher than growth in kinetin $(\mathrm{t}=0.0171, \mathrm{df}=6, \mathrm{p}<0.05)$, indole butyric acid $(\mathrm{t}=0.0455$, $\mathrm{df}=6, \mathrm{p}<0.05)$, mixture $(\mathrm{t}=0.0293, \mathrm{df}=6, \mathrm{p}<0.05)$ and control $(\mathrm{t}=0.0359, \mathrm{df}=6, \mathrm{p}<0.05)($ Table-11). 
Table $11 \mathrm{t}$-values showing the effect of $10 \mathrm{ppm}$ concentration of different growth regulators on mycelial growth of L. squarrosulus.

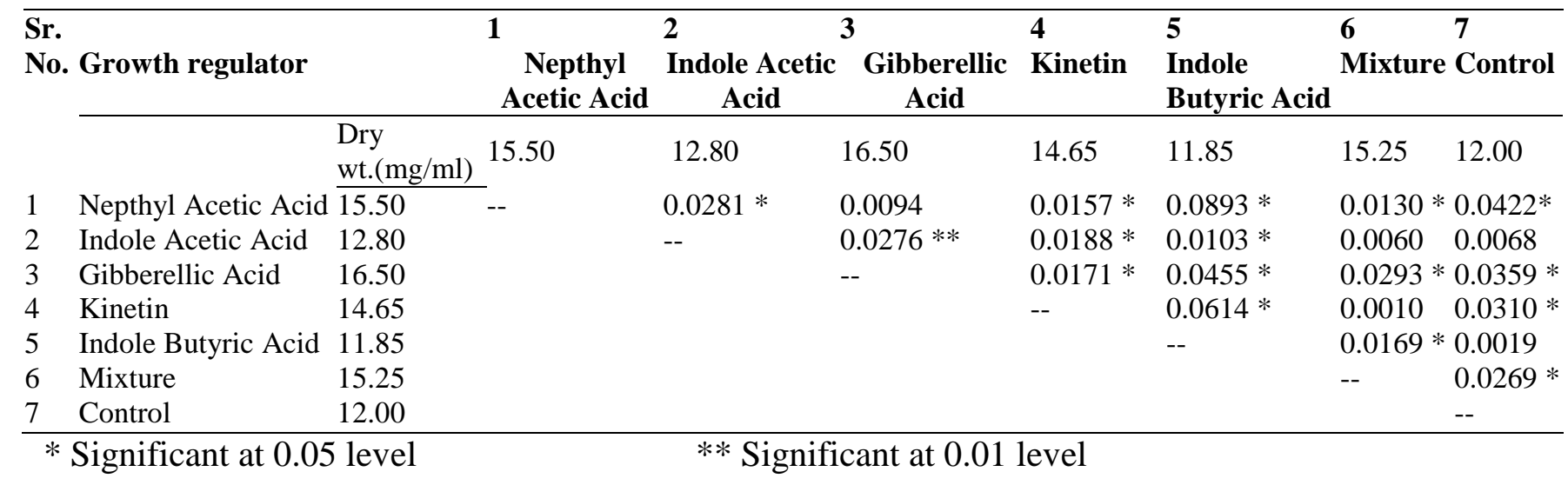

Next best vegetative growth was recorded in nepthyl acetic acid which was significantly higher than growth in indole acetic acid $(\mathrm{t}=0.0281, \mathrm{df}=6, \mathrm{p}<0.05)$, kinetin $(\mathrm{t}=0.0157, \mathrm{df}=6, \mathrm{p}<0.05)$, indole butyric acid $(\mathrm{t}=0.0893, \mathrm{df}=6, \mathrm{p}<0.05)$, mixture $(\mathrm{t}=0.0130, \mathrm{df}=6, \mathrm{p}<0.05)$ and control $(\mathrm{t}=0.0422, \mathrm{df}=6$, $\mathrm{p}<0.05)$. In comparison the growth with gibberellic acid was found to be non significant. Least vegetative growth was recorded in indole butyric acid (Table-11).

Maximum vegetative growth at $15 \mathrm{ppm}$ concentration was recorded in gibberellic acid which was significantly higher than growth in kinetin $(\mathrm{t}=0.0225, \mathrm{df}=6, \mathrm{p}<0.05)$, indole butyric acid ( $\mathrm{t}=0.0619$, $\mathrm{df}=6, \mathrm{p}<0.05)$, mixture $(\mathrm{t}=0.0279, \mathrm{df}=6, \mathrm{p}<0.05)$ and control $(\mathrm{t}=0.0504, \mathrm{df}=6, \mathrm{p}<0.05)($ Table-12).

Next maximum vegetative growth was recorded in nepthyl acetic acid which was significantly higher than growth in indole acetic acid $(\mathrm{t}=0.0491, \mathrm{df}=6, \mathrm{p}<0.05)$, gibberellic acid $(\mathrm{t}=0.0132, \mathrm{df}=6$, $\mathrm{p}<0.05)$, indole butyric acid $(\mathrm{t}=0.0468, \mathrm{df}=6, \mathrm{p}<0.05)$, and control $(\mathrm{t}=0.0391, \mathrm{df}=6, \mathrm{p}<0.05)$. In comparison the growth in kinetin and mixture was found to be non significant. Amongst the different growth regulators used, least vegetative growth was recorded in indole butyric acid (Table-12).

Maximum vegetative growth at $20 \mathrm{ppm}$ concentration was recorded in gibberellic acid which was significantly higher than growth in kinetin $(\mathrm{t}=0.0224, \mathrm{df}=6, \mathrm{p}<0.05)$, indole butyric acid $(\mathrm{t}=0.0410$, $\mathrm{df}=6, \mathrm{p}<0.05)$, mixture $(\mathrm{t}=0.0259, \mathrm{df}=6, \mathrm{p}<0.05)$ and control $(\mathrm{t}=0.0535, \mathrm{df}=6, \mathrm{p}<0.05)$ (Table-11). Next best vegetative growth was recorded in nepthyl acetic acid which was significantly higher than growth in indole acetic acid $(\mathrm{t}=0.0411, \mathrm{df}=6, \mathrm{p}<0.05)$, gibberellic acid $(\mathrm{t}=0.0141, \mathrm{df}=6, \mathrm{p}<0.05)$, kinetin $(\mathrm{t}=0.0106, \mathrm{df}=6, \mathrm{p}<0.05)$, indole butyric acid $(\mathrm{t}=0.0353, \mathrm{df}=6, \mathrm{p}<0.05)$, mixture $(\mathrm{t}=0.0160, \mathrm{df}=6$, $\mathrm{p}<0.05)$ and control $(\mathrm{t}=0.0530, \mathrm{df}=6, \mathrm{p}<0.05)$. Least vegetative growth was recorded in indole butyric acid (Table -13).

\section{Discussion}

During the present investigation, for vegetative growth of L. squarrosulus nicotinic acid @ $15.5 \mathrm{mg} / \mathrm{ml}$ of concentration in basal medium gave best mycelial growth on dry weight basis. Wuyep et al. (2003) also employed malt extract agar incorporated with $0.0002 \% \mathrm{NaH}_{2} \mathrm{PO}_{4}, 0.001 \%$ $\mathrm{FeSO}_{4} .7 \mathrm{H}_{2} \mathrm{O}, 2.0 \%$ thiamine hydrochloride and $2 \% \mathrm{H}_{3} \mathrm{BO}_{3}$ for maintenance of culture of this mushroom. Atri et al. (2010) observed for maximum vegetative growth of L. connatus thiamine @ $0.01 \mathrm{mg} / 100 \mathrm{ml}$ of concentration gave best vegetative growth in basal medium on dry weight basis. Kaur and Lakhanpal (1995) reported maximum mycelial growth in case of Lentinula edodes in medium with 20 ppm of thiamine. Hiroe and Ikuda (1960) and Ishikawa (1967) have also reported thiamine stimulating best mycelial growth in case of Lentinula edodes. Out of the growth regulators employed, best mycelial growth on dry weight basis in L. connatus has been documented in 5 ppm 
Table 12 t-values showing the effect of $15 \mathrm{ppm}$ concentration of different growth regulators on mycelial growth of L. squarrosulus.

\begin{tabular}{|c|c|c|c|c|c|c|c|c|c|}
\hline $\begin{array}{l}\text { Sr. } \\
\text { No. }\end{array}$ & Growth regulator & & $\begin{array}{l}1 \\
\text { Nepthyl } \\
\text { Acetic Acid }\end{array}$ & $\begin{array}{l}2 \\
\text { Indole } \\
\text { Acetic Acid }\end{array}$ & $\begin{array}{l}3 \\
\text { Gibberellic } \\
\text { Acid }\end{array}$ & $\begin{array}{l}\mathbf{4} \\
\text { Kinetin }\end{array}$ & $\begin{array}{l}5 \\
\text { Indole Butyri } \\
\text { Acid }\end{array}$ & $\begin{array}{l}6 \\
\text { c Mixture }\end{array}$ & $\begin{array}{l}7 \\
\text { Control }\end{array}$ \\
\hline & & $\begin{array}{l}\text { Dry wt. } \\
\text { (mg/ml) }\end{array}$ & 15.9 & 13.25 & 16.75 & 15.30 & 12.20 & 15.60 & 12.15 \\
\hline 1 & Nepthyl Acetic Acid & 15.9 & -- & $0.0491 *$ & $0.0132 *$ & 0.0085 & $0.0468 *$ & 0.0058 & $0.0391 *$ \\
\hline 2 & Indole Acetic Acid & 13.25 & & -- & $0.0768 *$ & $0.0380 *$ & $0.0162 *$ & $0.0673 *$ & $0.0130 *$ \\
\hline 3 & Gibberellic Acid & 16.75 & & & -- & $0.0225 *$ & $0.0619 *$ & $0.0279 *$ & $0.0504 *$ \\
\hline 4 & Kinetin & 15.30 & & & & -- & $0.0392 *$ & $0.0132 *$ & $0.0329 *$ \\
\hline 5 & Indole Butyric Acid & 12.20 & & & & & -- & $0.0230 *$ & 0.0004 \\
\hline 6 & Mixture & 15.60 & & & & & & -- & 0.0010 \\
\hline 7 & Control & 12.15 & & & & & & & -- \\
\hline
\end{tabular}

* Significant at 0.05 level

Table 13 t-values showing the effect of $20 \mathrm{ppm}$ concentration of different growth regulators on mycelial growth of L. squarrosulus.

\begin{tabular}{|c|c|c|c|c|c|c|c|c|c|}
\hline \multirow{3}{*}{\multicolumn{2}{|c|}{$\begin{array}{l}\text { Sr. } \\
\text { No. Growth regulator }\end{array}$}} & & 1 & 2 & 3 & \multirow{2}{*}{$\begin{array}{l}4 \\
\text { Kinetin }\end{array}$} & 5 & \multirow{2}{*}{$\begin{array}{l}6 \\
\text { Mixture }\end{array}$} & \multirow{2}{*}{$\begin{array}{l}7 \\
\text { Control }\end{array}$} \\
\hline & & & $\begin{array}{l}\text { Nepthyl Acetic } \\
\text { Acid }\end{array}$ & $\begin{array}{l}\text { Indole A } \\
\text { Acid }\end{array}$ & $\begin{array}{l}\text { icGibberellic } \\
\text { Acid }\end{array}$ & & Butyric Acid & & \\
\hline & & $\begin{array}{l}\text { Dry wt. } \\
(\mathrm{mg} / \mathrm{ml})\end{array}$ & 16.20 & 13.55 & 17.55 & 15.55 & 12.45 & 15.90 & 12.30 \\
\hline 1 & Nepthyl Acetic Acid & 16.20 & -- & $0.0411 *$ & $0.0141 *$ & $0.0106 *$ & $0.0353 *$ & $0.0160 *$ & $0.0530 *$ \\
\hline 2 & Indole Acetic Acid & 13.55 & & -- & $0.0438 *$ & $0.0371 *$ & $0.0107 *$ & $0.0173 *$ & $0.0184 *$ \\
\hline 3 & Gibberellic Acid & 17.55 & & & -- & $0.0224 *$ & $0.0410 *$ & $0.0259 *$ & $0.0535 *$ \\
\hline 4 & Kinetin & 15.55 & & & & -- & $0.0309 *$ & $0.0180 *$ & $0.0502 *$ \\
\hline 5 & Indole Butyric Acid & 12.45 & & & & & -- & $0.0369 *$ & 0.0013 \\
\hline 6 & Mixture & 15.90 & & & & & & -- & 0.0010 \\
\hline 7 & Control & 12.30 & & & & & & & -- \\
\hline
\end{tabular}

* Significant at 0.05 level

concentration of indole -3- butyric acid in the basal medium. Kaur and Lakhanpal (1995) reported maximum mycelial growth of Lentinula edodes in gibberellic acid.

Manjunathan and Kaviyarasan (2010) observed that thiamine gave best among the vitamins followed by biotin and togoferrol. Lander (1954) also found that thiamine stimulates mycelial growth of Cercospora arachidicola Hori in liquid culture. Madunagu (1988) also observed that thiamine is required for good growth in mushrooms. Luo (1993) reported that, different vitamins produce different effects on mycelial growth within a certain concentration range. Nolan (1970) observed that combined amino acids stimulate much growth than single amino acids. Adejoye et al. (2007) reported riboflavin and pyridoxine promoted the best growth of 85 and $100.45 \mathrm{mg} / 30 \mathrm{~cm}$ in Schizophyllum commune There are no such studies available for comparing the results in L. squarrosulus, however Treschow (1944) documented the requirement of biotin and thiamine for mycelial growth in case of Agaricus bisporus.

In case of Lentinula edodes, Han et al. (1981) obtained the best mycelial growth with $10 \mathrm{mg} / \mathrm{l}$ of gibberellic acid. Chang and Miles (1989) studied the effect of indole-3-acetic acid on the mycelial growth in Lentinula edodes, and reported that in all the concentrations of indole-3-acetic acid, growth was higher in comparison to control. Sladky and Tichy (1974) applied indole-3-acetic acid, gibberellic acid and kinetin to Lentinus trigrinus cultivated on small "Cellulose cylinders" and results indicated 
that solution of 100 ppm IAA and 400 ppm gibberellic acid increased mean sporophore size by $25 \%$ and $16 \%$, respectively. In present study at $20 \mathrm{ppm}$ concentration maximum mycelial growth of 17.55 $\mathrm{mg} / \mathrm{ml}$ was recorded in gibberellic acid and least mycelial growth of $12.45 \mathrm{mg} / \mathrm{ml}$ was there in Indole3-butyric acid. So far there is no work of this type by any worker on L. squarrosulus.

\section{Acknowledgements}

Thanks are due to Head Department of Botany Punjabi University Patiala for providing laboratory facilities and to U.G.C. for grant under ASIST and DRS (SAP III) Programs.

\section{References}

Adejoye OD, Adebayo-Tayo BC, Ogunjobi AA, Afolabi OO. 2007 - Physicochemical Studies on Schizophyllum commune (Fries) a Nigerian Edible Fungus. World Applied Sciences Journal 2 (1), 73-76.

Atri NS, Kaur A, Kaur H. 2005- Wild Mushrooms - Collection and identification. In: Frontiers in Mushroom Biotechnology (RD Rai, RC. Upadhyay and SR Sharma, eds.) National Research Center for Mushrooms, Chambaghat, Solan, 9-26.

Atri NS, Kumari D, Sharma SK. 2010 - Effect of vitamins and growth regulators on the vegetative growth of Lentinus connatus Berk. Indian Journal of Mushrooms 28(1\&2), 63-69.

Chang ST, Miles PG. 1987 - Historical record of the early cultivation of Lentinus in China. Mushroom Journal of Tropics. 7, 31-37.

Han YH, Yeng WT, Cheng LC, Chang S. 1981- Physiology and ecology of Lentinus edodes (Berk.) Sing. Mushroom Science. 11, 623 - 658.

Hiroe I, Ikuda J. 1960 - Physiological and biological studies on Lentinus edodes (Berk.) Singer. IV. Studies on nutritional physiology. Tottori Society of Agricultural Science. 12, 7-12.

Ishikawa H. 1967 - Physiological and ecological studies on Lentinus edodes (Berk.) Singer. Journal Agric lab (jpn.).8, 1-57.

Kaur MJ, Lakhanpal TN. 1995 - Effect of nutrient elements, vitamins and growth regulators on the vegetative growth of Lentinus edodes. Mushroom Research 4, 11-14.

Kirk PM, Cannon PF, Minter PF, Stalpers JA. 2008 - Ainsworth Bisby's Dictionary of Fungi $\left(10^{\text {th }}\right.$ ed.) CAB International Wallingford, Oxon, OX10, 8DE., UK.

Lander KE. 1964 - Growth of Cercospora arachidicola in a glucose phosphate, asparagine-thiamine agar medium. Phytopathology, 54, 1236-1240.

Luo XC. 1993 - Biology of artificial log cultivation of Auricularia mushroom. In: Chang, S.T., Buswell JA, Siu-wai Chiu (eds) Mushroom Biology and Mushroom Cultivation. Chinese University Press, Hong Kong, pp: 370.

Madunagu BE. 1988 - Collection and studies on the cultivation of Pleurotus squarrosulus (Mont) Sing. Nigerian Journal of Science, 22, 51-55.

Manjunathan J, Kaviyarasan V. 2010 - Studies on the growth requirements of Lentinus tuberregium (Fr.), An edible mushroom. Middle-East Journal of Scientific Research 5 (2), 81-85.

Nolan RA. 1970 - The Phycomycetes catenaries anguillulae growth requirements. Journal General Microbiology, 60, 167-168.

Pegler DN. 1977 - A preliminary Agaric flora of East Africa. Her Majesty's Stationary Office, London, pp.41- 42.

Sladky Z, Tichy V. 1974 - Stimulation of the formation of fruiting bodies of the fungus Lentinus tigrinus (Bull.) Fr. by growth regulators. Biologia Plantarum (Prague), 16(6), 436-443.

Treschow C. 1944 - Nutrition of the cultivated mushroom. Dansk Botanisk Arkiv. 11, $1-180$. 
Wuyep PA, Khan AU, Nok AJ. 2003 - Production and regulation of lignin degrading enzymes from Lentinus squarrosulus (Mont.) Singer and Psathyrella atroumbonata Pegler. African Journal of Biotechnology, 2(11), 444-447. 